Y. Teranishi

Nagoya Math. J.

Vol. 98 (1985), 139-156

\title{
RELATIVE INVARIANTS AND $b$-FUNCTIONS OF PREHOMOGENEOUS VECTOR SPACES
}

\author{
$\left(G \times G L\left(d_{1}, \cdots, d_{r}\right), \tilde{\rho}_{1}, M(n, C)\right)$
}

\section{YASUO TERANISHI}

\section{Introduction}

Let $G$ be a connected linear algebraic group, $\rho$ a rational representation of $G$ on a finite-dimensional vector space $V$, all defined over $C$.

A polynomial $f(x)$ on $V$ is called a relative invariant, if there exists a rational character $\chi: G \rightarrow C^{\times}$satisfying

$$
f(\rho(g) \cdot x)=\chi(g) f(x), \quad \text { for any } g \in G \text { and } x \in V .
$$

The triplet $(G, \rho, V)$ is called a prehomogeneous vector space (abbrev. P.V.), if there exists a proper algebraic subset $S$ of $V$ such that $V-S$ is a single $G$-orbit. The algebraic set $S$ is called the singular set of $(G, \rho, V)$ and any point in $V-S$ is called a generic point of $(G, \rho, V)$.

Let $G L\left(d_{1}, \cdots, d_{r}\right)$ be a parabolic subgroup of the general linear group $G L(n, C)$ defined by (1.1) in Section 1, $\rho: G \rightarrow G L(n, C)$ be an $n$-dimensional representation of $G$. In this paper, we shall be concerned with the triplet $\left(G \times G L\left(d_{1}, \cdots, d_{r}\right), \tilde{\rho}_{1}, M(n, C)\right)$, where $\tilde{\rho}_{1}$ is defined by

$$
\rho_{1}(g, a) x=\rho(g) x a^{-1} \quad\left((g, a) \in G \times G L\left(d_{1}, \cdots, d_{r}\right), x \in M(n, C)\right) .
$$

Assume that $\left(G \times G L\left(d_{1}, \cdots, d_{r}\right), \tilde{\rho}_{1}, M(n, C)\right)$ is a P.V. We shall introduce the $b$-function of $\left(G \times G L\left(d_{1}, \cdots, d_{r}\right), \tilde{\rho}_{1}, M(n, C)\right)$, after M. Sato, in Section 3. Theorem 3.1 gives an explicit form of the $b$-function. In Section 4, we shall be concerned with triplets $\left\{\left(G \times B_{n}, \tilde{\rho}_{1}, M(n, C)\right)\right\}$ where $G$ is a semi-simple connected linear algebraic group, $B_{n}$ is the upper triangular group and $\rho$ is an irreducible representation on an $n$-dimensional vector space $V$. We shall determine all prehomogeneous vector space $\left\{\left(G \times B_{n}, \tilde{\rho}_{1}, M(n, C)\right\}\right.$, and construct their relative invariants.

Received May 7, 1984. 
Notations. $C, C^{\times}$and $Z_{+}$are the complex number field, the group of non-zero complex numbers and the set of non-negative integers, respectively. $\mathrm{G} L(n, C), B_{n}$ and $B_{n}^{-}$are the complex general linear group, the complex upper triangular group and the complex lower triangular group.

\section{§1. A generalization of castling transform}

Let $G$ be a connected linear algebraic group, $V$ an $m$-dimensional vector space, and $\rho$ a rational representation of $G$ on $V$, all defined over the complex number field $C$. By choosing a basis of $V$, we may identify $V$ with $C^{m}$. Let $d_{1}, \cdots, d_{r}$ be positive integers and set

$$
n=d_{1}+\cdots+d_{r} \text { and } d^{(i)}=d_{1}+\cdots+d_{i} \quad(1 \leqq i \leqq r) .
$$

We denote by $G L\left(d_{1}, \cdots, d_{r}\right)$ the parabolic subgroup of the general linear group $G L(n, C)$ consists of all matrices of the form

$$
g=\left(\begin{array}{cccc}
g_{11} & g_{12} \cdots & g_{1 r} \\
0 & g_{22} & \cdots & g_{2 r} \\
\vdots & & \vdots \\
0 & 0 & \cdots & g_{r r}
\end{array}\right)
$$

where $g_{i i} \in G L\left(d_{i}, C\right)(1 \leqq i \leqq r)$.

We may identify the vector space $\oplus^{n} V$ with the vector space $M(m, n, C)$ consists of all $m$ by $n$ matrices and identify the vector space $M(m, n, C)$ with it's dual vector space by the inner product

$$
(x, y)=\operatorname{Tr}^{t} y \cdot x \quad(x, y \in M(m, n, C)) .
$$

Let $\tilde{\rho}_{1}, \tilde{\rho}_{2}, \tilde{\rho}_{1}^{*}$ and $\tilde{\rho}_{2}^{*}$ denote representations lof $G \times{ }_{*}^{:} G L\left(d_{1}, \cdots, d_{r}\right)$ on $M(m, n, C)$ defined as follows:

$$
\begin{aligned}
& \tilde{\rho}_{1}(g, a) x=\rho(g) x a^{-1} \\
& \tilde{\rho}_{2}(g, a) x=\rho(g) x^{t} a \\
& \tilde{\rho}_{1}^{*}(g, a) x={ }^{t} \rho(g)^{-1} x^{t} a \\
& \tilde{\rho}_{2}^{*}(g, a) x={ }^{t} \rho(g)^{-1} x a^{-1}
\end{aligned}
$$

where $g \in G$ and $a \in G L\left(d_{1}, \cdots, d_{r}\right)$.

LEMMA 1.1. The following conditions are equivalent.

(i) The triplet $\left(G \times G L\left(d_{1}, \cdots, d_{r}\right), \tilde{\rho}_{1}, M(m, n, C)\right)$ is a P.V.

(ii) The triplet $\left(G \times G L\left(d_{r}, \cdots, d_{1}\right), \tilde{\rho}_{2}, M(m, n, C)\right)$ is a P.V. 
Proof. Put $A=\left[\cdot^{\cdot} \cdot^{1}\right] \in G L(n, C)$, then it is easy to check that $x_{0} \in M(m, n, C)$ is a generic point of the triplet $\left(G \times G L\left(d_{1}, \cdots, d_{r}\right), \tilde{\rho}_{1}\right.$, $M(m, n, C))$ if and only if $x_{0} \cdot A$ is a generic point of $\left(G \times G L\left(d_{1}, \cdots, d_{r}\right)\right.$, $\left.\tilde{\rho}_{2}, M(m, n, C)\right)$.

LEMMA 1.2. There exists a one-to-one correspondence between relative invariants of $\left(G \times G L\left(d_{1}, \cdots, d_{r}\right), \tilde{\rho}_{1}, M(m, n, C)\right)$ and $\left(G \times G L\left(d_{r}, \cdots, d_{1}\right)\right.$, $\left.\tilde{\rho}_{2}, M(m, n, C)\right)$.

Proof. For a polynomial $f(x)$ on $M(m, n, C)$, define the polynomial $\Phi(f)$ by

$$
\Phi(f)(x)=f(x \cdot A) .
$$

Then the mapping $f \mapsto \Phi(f)$ gives a one-to-one correspondence between relative invariants.

Q.E.D.

LEMMA 1.3. When $m>n$, the following conditions are equivalent.

(i) The triplet $\left(G \times G L\left(d_{1}, \cdots, d_{r}\right), \tilde{\rho}_{1}, M(m, n, C)\right)$ is a P.V.

(ii) The triplet $\left(G \times G L\left(m-n, d_{r}, \cdots, d_{2}\right), \tilde{\rho}_{2}^{*}, M\left(m, m-d_{1}, C\right)\right)$ is a P.V.

Proof. For a matrix $x$ in $M(m, n, C)$, denote by $x^{i}$ the $i$-th column vector of $x(1 \leqq i \leqq n)$. Let $W$ denote an algebraic variety whose points are matrices $x$ in $M(m, n, C)$ such that column vectors $x^{1}, x^{2}, \cdots$ and $x^{n}$ are linearly independent. Then the group $G \times G L\left(d_{1}, \cdots, d_{r}\right)$ acts on $W$, and $\left(G \times G L\left(d_{1}, \cdots, d_{r}\right), W\right)$ has an open orbit if and only if the triplet $\left(G \times G L\left(d_{1}, \cdots, d_{r}\right), \tilde{\rho}_{1}, M(m, n, C)\right)$ is a P.V., since the Zariski closure of $W$ is $M(m, n, C)$. Let $\operatorname{Flag}\left(d_{1}, \cdots, d_{r}\right)$ be the flag variety defined by

$$
\operatorname{Flag}\left(d_{1}, \cdots, d_{r}\right)=\left\{\begin{array}{ll}
\left(V_{1}, V_{2}, \cdots, V_{r}\right) ; & \begin{array}{l}
V_{i} \in \operatorname{Grass}_{d_{1}+\cdots+d_{i}}\left(C^{m}\right) \text { and } \\
V_{1} \subset V_{2} \subset \cdots \subset V_{r}
\end{array}
\end{array}\right\}
$$

where $\operatorname{Grass}_{d}\left(C^{m}\right)$ is the Grassmann variety consists of all $d$-dimensional subspaces of $C^{m}$.

For a matrix $x$ in the variety $W$, let $\mu(x)$ denote the flag $\left(V_{1}, \cdots, V_{n}\right)$ in Flag $\left(d_{1}, \cdots, d_{r}\right)$ such that $V_{i}$ is the subspace of $C^{m}$ spanned by the flrst $d_{1}+\cdots+d_{r}$ column vectors of the matrix $x(1 \leqq i \leqq r)$. Then the mapping $\mu: W \mapsto \operatorname{Flag}\left(d_{1}, \cdots, d_{r}\right)$ is surjective, $G \times G L\left(d_{1}, \cdots, d_{r}\right)$ equivalent 
morphism. Since $G L\left(d_{1}, \cdots, d_{r}\right)$ acts on Flag $\left(d_{1}, \cdots, d_{r}\right)$ trivially and it acts on each fibre homogeneously, the triplet $\left(G \times G L\left(d_{1}, \cdots, d_{r}\right), \tilde{\rho}_{1}\right.$, $M(m, n, C))$ is a P.V. if and only if $\operatorname{Flag}\left(d_{1}, \cdots, d_{r}\right)$ is $G$-prehomogeneous.

For a flag $\left(V_{1}, V_{2}, \cdots, V_{r}\right)$ in $\operatorname{Flag}\left(d_{1}, \cdots, d_{r}\right)$, let $\left(\tilde{V}_{1}, \tilde{V}_{2}, \cdots, \tilde{V}_{r}\right)$ be the flag in Flag $\left(m-n, d_{r}, \cdots, d_{2}\right)$ defined by

$$
\tilde{V}_{i}=\left\{y \in M(m, n, C) \mid(y, x)=0 \text { for any } x \text { in } V_{r-i+1}\right) .
$$

Then $G$ acts on the flag variety $\operatorname{Flag}\left(m-n, d_{r}, \cdots, d_{2}\right)$ contragrediently and Flag $\left(m-n, d_{r}, \cdots, d_{2}\right)$ is $G$-prehomogeneous if and only if Flag $\left(d_{1}\right.$, $\left.\cdots, d_{r}\right)$ is $G$-prehomogeneous.

Since the triplet $\left(G \times G L\left(m-n, d_{r}, \cdots, d_{2}\right), \tilde{\rho}_{2}^{*}, M\left(m, m-d_{1}, C\right)\right)$ is a P.V. if and only if the flag variety $\operatorname{Flag}\left(m-n, d_{r}, \cdots, d_{2}\right)$ is $G$-prehomogeneous, we obtain our assertion.

Q.E.D.

Remark. This construction is a natural generalization of the castling transform in the theory of prehomogeneous vector space [2].

LEMMA 1.4. When $m>n$, there is a one-to-one correspondence between relative invariants of the triplet $\left(G \times G L\left(d_{1}, \cdots, d_{r}\right), \tilde{\rho}_{1}, M(m, n, C)\right)$ and relative invariants of the triplet $\left(G \times G L\left(m-n, d_{r}, \cdots, d_{2}\right), \tilde{\rho}_{2}, M\left(m, m-d_{1}\right.\right.$, C)).

Proof. Let $f\left(x^{1}, \cdots, x^{n}\right)$ be a relative invariant of the triplet $(G \times G L$ $\left.\left(d_{1}, \cdots, d_{r}\right), \tilde{\rho}_{1}, M(m, n, C)\right)$, where $x^{i}$ is the $i$-th column vector of an $m \times n$ matrix $x(1 \leqq i \leqq n)$. For $x=\left(x^{1}, \cdots, x^{n}\right) \in M(m, n, C)$, put

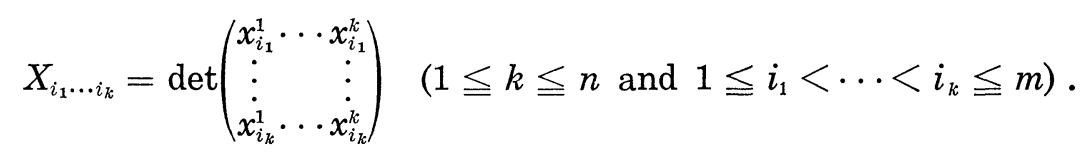

Then by the first main theorem for the group $G L\left(d_{1}, \cdots, d_{r}\right)$, there exists a polynomial $F$ satisfying

$$
\begin{gathered}
f\left(x^{1}, \cdots, x^{n}\right)=F\left(X_{i_{1} \cdots i_{d_{1}}}, X_{j_{1} \cdots j_{d_{1}+d_{2}}}, \cdots, X_{k_{1} \cdots k_{d_{1}+\cdots+d_{r}}}\right) \\
\left(1 \leqq i_{1}, \cdots, i_{d_{1}}, j_{1}, \cdots, j_{d_{1}+d_{2}}, k_{1}, \cdots, k_{d_{1}+\cdots+d_{r}} \leqq m\right),
\end{gathered}
$$

since $f(x)$ is a relative invariant of the group $G L\left(d_{1}, \cdots, d_{r}\right)$.

For $x=\left(x^{1}, \cdots, x^{n}\right)$ in $M(m, n, C)$, let $\omega_{k}=x^{1} \wedge \cdots \wedge x^{k}$ and, for $\tilde{x}=$ $\left(\tilde{x}^{1}, \cdots, \tilde{x}^{m-d_{1}}\right)$ in $M\left(m, m-d_{1}, C\right)$, let $\tilde{\omega}_{k}=\tilde{x}^{1} \wedge \cdots \wedge \tilde{\omega}^{m-k}$ where $k \epsilon$ $\left\{d_{1}, d_{1}+d_{2}, \cdots, d_{1}+\cdots+d_{r}\right\}$.

Then it follows that 


$$
\begin{aligned}
& \omega_{k}=\sum_{i_{1}<\cdots<i_{k}} X_{i_{1} \cdots i_{k}} e_{i_{1}} \wedge \cdots \wedge e_{i_{k}}, \\
& \tilde{\omega}_{k}=\sum_{j_{1}<\cdots<j_{m-k}} \tilde{X}_{j_{1} \cdots j_{m-k}} e_{j_{1}} \wedge \cdots \wedge e_{j_{m-k}}\left(e_{i}={ }^{t}(0 \cdots 1 \cdots 0), 1 \leqq i \leqq m\right),
\end{aligned}
$$

and we have

$$
\sum_{i_{1}<\cdots<i_{k}} \operatorname{sgn}\left(\begin{array}{l}
1 \cdots k, k+1 \cdots m \\
i_{1} \cdots i_{k}, j_{1} \cdots j_{m-k}
\end{array}\right) X_{i_{1} \cdots i_{k}} \tilde{X}_{j_{1} \cdots j_{m-k}}=\operatorname{det}\left(x^{1}, \cdots x^{k}, \tilde{x}^{1}, \cdots \tilde{x}^{m-k}\right)
$$

where

$$
\operatorname{sgn}\left(\begin{array}{l}
1 \cdots k, k+1 \cdots m \\
i_{1} \cdots i_{k}, j_{1} \cdots j_{m-k}
\end{array}\right)
$$

denotes the signature of the permutation $\left(\begin{array}{c}1 \cdots k, k+1 \cdots m \\ i_{1} \cdots i_{k}, j_{1} \cdots j_{m-k}\end{array}\right)$ if

$$
\left\{i_{1} \cdots i_{k}, j_{1} \cdots j_{m-k}\right\}=\{1,2 \cdots m\}
$$

and zero, if otherwise.

Thus if we put

$$
X_{i_{1} \cdots i_{k}}^{\prime}=\operatorname{sgn}\left(\begin{array}{l}
1 \cdots k, k+1 \cdots m \\
i_{1} \cdots i_{k}, j_{1} \cdots j_{m-k}
\end{array}\right) \tilde{X}_{j_{1} \cdots j_{m-k}}
$$

we have

$$
\sum_{i_{1}<\cdots<i_{k}} X_{\imath_{1} \cdots i_{k}} X_{i_{1} \cdots i_{k}}^{\prime}=\operatorname{det}\left(x^{1}, \cdots, x^{k}, \tilde{x}^{1}, \cdots, \tilde{x}^{m-k}\right) .
$$

We define a polynomial $\tilde{f}(\tilde{x})$ on $M\left(m, m-d_{1}, C\right)$ by

$$
\tilde{f}(\tilde{x})=F\left(X_{i_{1} \cdots i_{d_{1}}}^{\prime} X_{j_{1} \cdots j_{d_{1}+d_{2}}}^{\prime} \cdots X_{k_{1} \cdots k_{d}+\cdots+d_{r}}^{\prime}\right) .
$$

Then $\tilde{f}$ is a relative invariant of the triplet $\left(G \times G L\left(m-n, d_{r} \cdots d_{2}\right), \tilde{\rho}_{2}^{*}\right.$, $M\left(m, m-d_{1} . C\right)$ ), and the mapping $f \mapsto \tilde{f}$ gives a one-to-one correspondence between relative invariants of them.

Q.E.D.

Remark. From the construction, $f$ is irreducible if and only if $\tilde{f}$ is irreducible.

By Lemma 1.1 1.4, we have the following proposition.

Proposition 1.1. When $m>n$, the following 4 conditions are equivalent and there are one-to-one correspondences among relative invariants of them.

(1) The triplet $\left(G \times G L\left(d_{1}, \cdots, d_{r}\right), \tilde{\rho}_{1}, M(m, n, C)\right)$ is a P.V.

(2) The triplet $\left(G \times G L\left(d_{r}, \cdots, d_{1}\right), \tilde{\rho}_{2}, M(m, n, C)\right)$ is a P.V. 
(3) The triplet $\left(G \times G L\left(d_{2}, \cdots, d_{r}, m-n\right)\right.$, $\tilde{\rho}_{1}^{*}, M\left(m, m-d_{1}, C\right)$ is a P.V.

(4) The triplet $\left(G \times G L\left(m-n, d_{r}, \cdots, d_{2}\right), \tilde{\rho}_{2}^{*}, M\left(m, m-d_{1}, C\right)\right)$ is a P.V.

The following two propositions are shown in a similar manner.

Proposition 1.2. Let $G$ be a connected linear algebraic group and $\rho$ a linear representation of $G$ on an $n$-dimensional vector space. Then the following 4 conditions are equivalent and there are one-to-one correspondences among their relative invariants

(1) The triplet $\left(G \times G L\left(d_{1}, \cdots, d_{r}\right), \tilde{\rho}_{1}, M(n, C)\right)$ is a P.V.

(2) The triplet $\left(G \times G L\left(d_{r}, \cdots, d_{1}\right), \tilde{\rho}_{2}, M(n, C)\right)$ is a P.V.

(3) The triplet $\left(G \times G L\left(d_{1}, \cdots, d_{r}\right), \tilde{\rho}_{1}^{*}, M(n, C)\right)$ is a $P . V$.

(4) The triplet $\left(G \times G L\left(d_{r}, \cdots, d_{1}\right), \tilde{\rho}_{2}^{*}, M(n, C)\right)$ is a P.V.

Proposition 1.3. When $m>n$, the following 4 conditions are equivalent.

(1) The triplet $\left(G \times G L\left(d_{1}, \cdots, d_{r}\right), \tilde{\rho}_{1}, M(m, n, C)\right)$ is a P.V.

(2) The triplet $\left(G \times G L\left(d_{r}, \cdots, d_{1}\right), \tilde{\rho}_{2}, M(m, n, C)\right)$ is a P.V.

(3) The triplet $\left(G \times G L\left(d_{1}, \cdots, d_{r}, m-n\right), \tilde{\rho}_{1}^{*}, M(m, C)\right)$ is a P.V.

(4) The triplet $\left(G \times G L\left(m-n, d_{r}, \cdots, d_{1}\right)\right.$, $\left.\tilde{\rho}_{2}^{*}, M(m, C)\right)$ is a P.V.

CoRollary. When $m>n$, the following conditions are equivalent.

(1) The triplet $\left(G \times G L\left(d_{1}, \cdots, d_{r}\right), \tilde{\rho}_{1}, M(m, n, C)\right)$ is a P.V.

(2) The triplet $\left(G \times G L\left(d_{1}, \cdots, d_{r}, m-n\right), \tilde{\rho}_{1}, M(m, C)\right.$ is a P.V.

Let $G$ be a connected linear algebraic group, $\rho$ a representation on an $n$-dimensional vector space $V$. Then, by Proposition 1.1, triplet $(G \times G L(1)$, $\rho \otimes \square, V)$ is a P.V. if and only if $\left(G \times G L(1, n-1), \tilde{\rho}_{1}, M(n, C)\right)$ is a P.V. We shall devote ourselves to investigate triples $\left(G \times G L\left(d_{1}, \cdots, d_{r}\right), \tilde{\rho}_{1}\right.$, $M(n, C))$.

\section{§2. Relative invariants}

A sequence $\lambda=\left(\lambda_{1}, \lambda_{2}, \cdots, \lambda_{n}\right)$ of non-negative integers in decreasing order;

$$
\lambda_{1} \geq \lambda_{2} \geq \cdots \geq \lambda_{n}
$$

is called a partition, and the sum $|\lambda|=\lambda_{1}+\cdots+\lambda_{n}$ is called the weight of $\lambda$. 
For a partition $\lambda$, we denote by $V_{\lambda}$ the vector space consists of all polynomials $f(x)$ on the vector space $M(n, C)$ such that $f(x)$ satisfies; for any matrix $t$ in the group $B_{n}$,

$$
f(x \cdot t)=t_{1}^{\lambda_{1}} t_{2}^{\lambda_{2}} \cdots t_{n}^{\lambda_{n}} f(x)
$$

where

$$
t=\left(\begin{array}{lll}
t_{1} & & * \\
& \ddots & \\
& & t_{n}
\end{array}\right) \in B_{n} .
$$

Let $f(x)$ be a polynomial on $M(n, C)$, and for any element $g$ in $G L(n, C)$, set;

$$
g \cdot f(x)=f\left(g^{-1} x\right) .
$$

Then by the mapping $f \mapsto g \cdot f$, the vector space $V_{\lambda}$ can be considered as a $G L(n, C)$-module. As is well known, $V_{2}$ is a irreducible $G L(n, C)$-module corresponding to the Young diagram $Y(\lambda)$ :

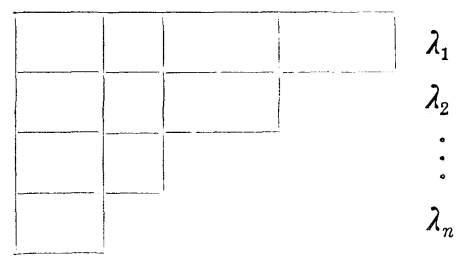

We set:

$$
X_{i_{1} \cdots i_{d}}^{\prime}=\operatorname{sgn}\left(\begin{array}{c}
1, \cdots, d, d+1, \cdots, n \\
i_{1}, \cdots, i_{d}, j_{1}, \cdots, j_{n-d}
\end{array}\right) \operatorname{det}\left(\begin{array}{c}
X_{j_{1}}^{d+1} \cdots X_{j_{1}}^{n} \\
\vdots \\
X_{j_{n-d}}^{d+1} \cdots \dot{X}_{j_{n-d}}^{n}
\end{array}\right)
$$

Let $f(x)$ be a relative invariant of the triplet $\left(G \times G L\left(d_{1}, \cdots, d_{r}\right)\right.$, $M(n, C)$. Then $f(x)$ has the form

$$
f(x)=F\left(\cdots, X_{i_{1} \cdots i_{d}(\nu)}, \cdots\right)(\operatorname{det} x)^{m_{r}},
$$

where $F$ is a homogeneous polynomial in $X_{i_{1} \cdots i_{d}(\nu)}\left(1 \leqq i_{1}, \cdots, i_{d}(\nu) \leqq n\right.$, $1 \leqq \nu<r$ ) and $m_{r}$ is a non-negative integer.

Denoting by $m_{\nu}$ the homogeneous degree of $F$ with respect to $X_{i_{1} \cdots i_{\tilde{U}}(\nu)}$, for each $\nu$, define a partition $\lambda=\left(\lambda_{1}, \cdots, \lambda_{n}\right)$ as follows:

$$
\begin{aligned}
& \lambda_{1}=\cdots=\lambda_{d^{(1)}}=m_{1}+\cdots+m_{r}, \\
& \lambda_{d^{(1)}+1}=\cdots=\lambda_{d^{(2)}}=m_{2}+\cdots+m_{r}, \\
& \cdots \cdots \\
& \lambda_{d^{(r-1)}+1}=\cdots=\lambda_{n}=m_{r} .
\end{aligned}
$$


Then the relative invariant $f(x)$ is contained in the vector space $V_{\lambda}$. Thus to a relative invariant $f(x)$, there corresponds the unique Young diagram $Y(\lambda)$ :

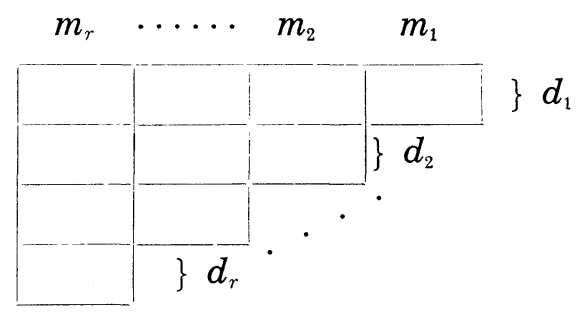

Let

$$
f^{*}(x)=(\operatorname{det} x)^{m_{r}} F\left(X_{\imath_{1} \cdots i_{d(1)}}, X_{j_{1} \cdots j_{d(2)}}, \cdots, X_{k_{1} \cdots k_{d(r-1)}}\right)
$$

be a relative invariant of the triplet $\left(G \times G L\left(d_{1}, \cdots, d_{r}\right), \tilde{\rho}_{1}, M(n, C)\right)$, and define the polynomial $f^{*}(x)$ by

$$
f^{*}(x)=(\operatorname{det} x)^{m_{r}} F\left(X_{\imath_{1} \cdots i_{d(1)}}^{\prime}, X_{j_{1} \cdots j_{d(2)}}^{\prime}, \cdots, X_{k_{1} \cdots k_{d(r-1)}}^{\prime}\right) .
$$

Then $f^{*}(x)$ is a relative invariant of the triplet $\left(G \times G L\left(d_{1}, \cdots, d_{r}\right), \tilde{\rho}_{1}^{*}\right.$, $M(n, C))$. Since the mapping $f \mapsto f^{*}$ is one-to-one, we have the following proposition.

Proposition 2.1. The mapping $f \mapsto f^{*}$ gives a one-to-one correspondence between the relative invariants of the triplets $\left(G \times G L\left(d_{1}, \cdots, d_{r}\right), \tilde{\rho}_{1}\right.$, $M(n, C))$ and $\left(G \times G L\left(d_{1}, \cdots, d_{r}\right), \tilde{\rho}_{1}^{*}, M(n, C)\right)$.

In general, let a triplet $(G, \rho, V)$ be a P.V., $S$ the singular set, $S_{1}, \cdots$, $S_{k}$ the irreducible components of $S$ with codimension one and $P_{1}, \cdots, P_{k}$ irreducible polynomials defining $S_{1}, \cdots, S_{k}$, respectively. It is known that the polynomials $P_{1}, \cdots, P_{k}$ are algebraically independent relative invariants and any relative invariant $P(x)$ is of the form.

$$
P(x)=c \cdot P_{1}(x)^{l_{1}} \ldots P_{k}(x)^{l_{k}}\left(c \in C,\left(l_{1}, \cdots, l_{k}\right) \in Z_{+}^{k+1}\right) .
$$

Polynomials $P_{1}, \cdots, P_{k}$ are determined up to constant factors, and the set $\left\{P_{1}, \cdots, P_{k}\right\}$ is called a complete system of irreducible relative invariants of $(G, \rho, V)$.

Let $\left(G \times G L\left(d_{1}, \cdots, d_{r}\right), \tilde{\rho}_{1}, M(n, C)\right)$ be a P.V., $\left\{P_{0}, \cdots, P_{k}\right\}$ a complete system of irreducible relative invariants of $\left(G \times G L\left(d_{1}, \cdots, d_{r}\right), \tilde{\rho}_{1}, M(n, C)\right)$. Then, by Proposition 1.2, the triplet $\left(G \times G L\left(d_{1}, \cdots, d_{r}\right), \tilde{\rho}_{1}^{*}, M(n, C)\right)$ is a P.V. It is easy to verify that the set $\left\{P_{0}^{*}, \cdots, P_{k}^{*}\right\}$ is a complete system of 
irreducible relative invariants of $\left(G \times G L\left(d_{1}, \cdots, d_{r}\right), \tilde{\rho}_{1}^{*}, M(n, C)\right)$. Denote by $\chi_{0}, \cdots, \chi_{k}$ the rational characters of the group $G \times G L\left(d_{1}, \cdots, d_{r}\right)$ corresponding to $P_{0}, \cdots, P_{k}$, respectively.

Since det $x$ is an irreducible relative invariant of $\left(G \times G L\left(d_{1}, \cdots, d_{r}\right)\right.$. $\left.\tilde{\rho}_{1}, M(n, C)\right)$, from now on, we set:

$$
P_{0}(x)=P_{0}^{*}(x)=\operatorname{det} x .
$$

We denote by $X_{\rho}\left(G \times G L\left(d_{1}, \cdots, d_{r}\right)\right)$ the group of rational characters corresponding to relative invariants of $\left(G \times G L\left(d_{1}, \cdots, d_{r}\right), \tilde{\rho}_{1}, M(n, C)\right)$. The group $X_{p}\left(G \times G L\left(d_{1}, \cdots, d_{r}\right)\right)$ is a free abelian group of rank $k+1$ generated by $\chi_{0}, \cdots, \chi_{k}$.

Denote by $Y\left(\lambda^{(0)}\right), \cdots, Y\left(\lambda^{(k)}\right)$ the Young diagrams corresponding to relative invariants $P_{0}, \cdots, P_{k}$, respectively. Since each $P_{i}$ is irreducible, the partition $\lambda^{(i)}$ is of the form

$$
\lambda^{(i)}=\left(\lambda_{1}^{(i)}, \lambda_{2}^{(i)}, \cdots, \lambda_{n-1}^{(i)}, 0\right) \quad(1 \leq i \leq k) .
$$

The Young diagram $Y\left(\lambda^{(0)}\right)$ is given by

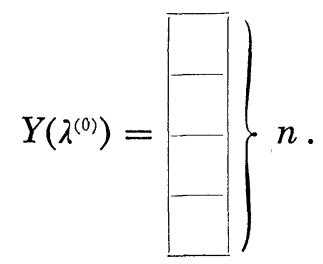

Denote by $\chi_{0}^{*}, \cdots, \chi_{k}^{*}$ the rational characters defined as follows:

$$
\chi_{0}^{*}=\chi_{0} \text { and } \chi_{i}^{*}=\chi_{i}^{-1} \cdot \chi_{0}^{\lambda_{1}^{(i)}} \quad(1 \leq i \leq k) .
$$

From the construction of the mapping $f \mapsto f^{*}$, it follows that:

$$
P_{i}^{*}\left(\tilde{\rho}_{1}^{*}(g) \cdot x\right)=\chi_{i}^{*}(g)^{-1} P_{i}^{*}(x) \quad(0 \leq i \leq k)
$$

where $g \in G \times G L\left(d_{1}, \cdots, d_{r}\right)$.

For a character $\chi$ in $X_{\rho}\left(G \times G L\left(d_{1}, \cdots, d_{r}\right)\right)$, let

$$
\delta(\chi)=\left(\delta(\chi)_{0}, \cdots, \delta(\chi)_{k}\right), \text { and } \delta^{*}(\chi)=\left(\delta^{*}(\chi)_{0}, \cdots, \delta^{*}(\chi)_{k}\right)
$$

be the elements in $Z^{k+1}$ such that

$$
\chi=\prod_{i=0}^{k} \chi_{i}^{\delta(x)_{i}}=\prod_{i=0}^{k} \chi_{i}^{* \delta *(x)_{i}}
$$

From (2.2), we have 


$$
\delta(\chi)_{0}=\delta^{*}(\chi)_{0}+\sum_{i=1}^{k} \lambda_{1}^{(i)} \delta^{*}(\chi)_{i}
$$

and

$$
\delta(\chi)_{i}=-\delta^{*}(\chi)_{i} \quad(1 \leq i \leq k)
$$

\section{$\S 3$. The $b$-functions}

For a rational character $\chi$ in $X_{\rho}\left(G \times G L\left(d_{1}, \cdots, d_{r}\right)\right)$, set

$$
P(x)^{x}=\prod_{i=0}^{k} P_{i}(x)^{\delta(x) i} \quad \text { and } \quad P^{*}(x)^{x}=\prod_{i=0}^{k} P_{i}^{*}(x)^{\delta^{*}(x) i} .
$$

If $\delta^{*}(\chi)_{i} \geq 0$ for all $i$ (i.e., $P^{*}(x)^{x}$ is a polynomial), we can introduce a partial differential operator $P^{*}(\operatorname{grad})^{x}$ in $C\left[\partial / \partial x_{i j}\right]$ such that

$$
P^{*}(\operatorname{grad})^{x} \exp \left(x, x^{*}\right)=P^{*}(x) \exp \left(x, x^{*}\right) .
$$

Similarly, if $P(x)^{x}$ is a polynomial, we can introduce $P(\operatorname{grad})^{x}$ in $C\left[\partial / \partial x_{i j}^{*}\right]$ such that

$$
P(\operatorname{grad})^{x} \exp \left(x, x^{*}\right)=P(x) \exp \left(x, x^{*}\right) .
$$

For $s=\left(s_{0}, \cdots, s_{k}\right) \in C^{k+1}$, set

$$
P^{s}=\prod_{i=0}^{k} P_{i}^{s_{t}} \quad \text { and } \quad P^{* s}=\prod_{i=0}^{k} P_{i}^{*^{s_{i}}}
$$

We consider $P^{s}$ (resp. $P^{*^{s}}$ ) as a function on the universal covering space of $M(n, C)-S$ (resp. $\left.M(n, C)-S^{*}\right)$.

LEMMA 3.1. (i) If $\delta^{*}(\chi)_{i} \geq 0$ for all $i$, there exists a polynomial $b_{\chi}(s)$ in $s=\left(s_{0}, \cdots, s_{k}\right)$ which satisfies, for all $s \in C^{k+1}$,

$$
P^{*}(\operatorname{grad})^{x} \cdot P^{s}(x)=b_{\chi}(s) P^{s-\delta(x)} .
$$

(ii) If $\delta(\chi)_{i} \geq 0$ for all $i$, there exists a polynomial $b_{x}^{*}(s)$ in $s=\left(s_{0}, \cdots, s_{k}\right)$ which satisfies, for all $s \in C^{k+1}$,

$$
P(\operatorname{grad})^{x} \cdot P^{* s}(x)=b_{x}^{*}(s) P^{* s-\delta *(x)} .
$$

Proof. Denoting by $F(x)$ the left hand side of (2.5), we have:

$$
F\left(\tilde{\rho}_{1}(g) \cdot x\right)=\chi^{-1}(g) \chi(g)^{s} F(x)
$$

and

$$
P\left(\tilde{\rho}_{1}(g) \cdot x\right)^{s-\delta(x)}=\chi^{-1}(g) \chi(g)^{s} P(x)^{s-\delta(\chi)} \quad\left(g \in G \times G L\left(d_{1}, \cdots, d_{r}\right)\right) .
$$


This shows that $P^{-s+\delta(x)} F(x)$ is an absolute invariant, and hence must be a constant $b_{x}(s)$ depending only upon $s$ and $\chi$. It is clear that $b_{\chi}(s)$ is a polynomial in $s$. The proof of the part (ii) is similar.

Q.E.D.

From the definitions of $b_{x}(s)$ and $b_{x}^{*}(s)$, it follows that:

(i) If $\chi$ and $\psi$ are characters in $X_{\rho}\left(G \times G L\left(d_{1}, \cdots, d_{r}\right)\right)$ such that $\delta^{*}(\chi)_{i} \geq 0$ and $\delta^{*}(\chi)_{i} \geq 0(0 \leq i \leq k)$, then

$$
b_{\chi \psi}(s)=b_{\chi}(s) b_{\psi}(s+\delta(\chi)) \text {. }
$$

(ii) If $\chi$ and $\psi$ are characters in $X_{\rho}\left(G \times G L\left(d_{1}, \cdots, d_{r}\right)\right)$ such that $\delta(\chi)_{i} \geq 0$ and $\delta(\chi)_{i} \geq 0(0 \leq i \leq k)$, then

$$
b_{\chi \psi}^{*}(s)=b_{\chi}^{*}(s) b_{\psi}^{*}\left(s+\delta^{*}(\chi)\right) .
$$

By the co-cycle properties of $b_{x}(s)$ and $b_{x}^{*}(s), b_{x}(s)$ and $b_{x}^{*}(s)$ can be defined for arbitrary character $\chi$ in $X_{\rho}\left(G \times G L\left(d_{1}, \cdots, d_{r}\right)\right)$.

Let $\lambda^{(1)}, \cdots, \lambda^{(k)}$ be the partitions corresponding to $P_{1}, \cdots, P_{k}$, respectively. For $s=\left(s_{0}, s_{1}, \cdots, s_{k}\right) \in C^{k+1}$, put

$$
\begin{aligned}
\gamma(s)= & \Gamma\left(s_{0}+s_{1} \lambda_{1}^{(1)}+\cdots+s_{k} \lambda_{1}^{(k)}+n\right) \\
& \times \Gamma\left(s_{0}+s_{1} \lambda_{2}^{(1)}+\cdots+s_{k} \lambda_{2}^{(k)}+n-1\right) \\
& \cdots \\
\times & \Gamma\left(s_{0}+s_{1} \lambda_{n-1}^{(1)}+\cdots+s_{k} \lambda_{n-1}^{(k)}+2\right) \\
\times & \Gamma\left(s_{0}+1\right),
\end{aligned}
$$

and

$$
\begin{aligned}
\gamma^{*}(s)= & \Gamma\left(s_{0}+s_{1} \lambda_{1}^{(1)}+\cdots+s_{k} \lambda_{1}^{(k)}+n\right) \\
& \times \Gamma\left(s_{0}+s_{1}\left(\lambda_{1}^{(1)}-\lambda_{n-1}^{(1)}\right)+\cdots+s_{k}\left(\lambda_{1}^{(k)}-\lambda_{n-1}^{(k)}\right)+n-1\right) \\
& \cdots \cdots \\
& \times \Gamma\left(s_{0}+s_{1}\left(\lambda_{1}^{(1)}-\lambda_{2}^{(1)}\right)+\cdots+s_{k}\left(\lambda_{1}^{(k)}-\lambda_{2}^{(k)}\right)+2\right) \\
& \times \Gamma\left(s_{0}+1\right) .
\end{aligned}
$$

Now we can state the main theorem of the present paper.

Theorem 3.1. Let $\left(G \times G L\left(d_{1}, \cdots, d_{r}\right), \tilde{\rho}_{1}, M(n, C)\right)$ be a P.V., $\left\{P_{0}, \cdots\right.$, $\left.P_{k}\right\}$ a complete system of relative invariants and $\lambda^{(1)}, \cdots, \lambda^{(r)}$ the partitions corresponding to $P_{1}, \cdots, P_{r}$. Then the b-functions $b_{x}(s)$ and $b_{x}^{*}(s)$ are given by

$$
b_{\chi}(s)=\frac{\gamma(s)}{\gamma(s-\delta(\chi))}
$$

and 


$$
b_{x}^{*}(s)=\frac{\gamma^{*}(s)}{\gamma^{*}\left(s-\delta^{*}(\chi)\right)}
$$

Proof. Let $B_{n}^{-}$denote the group consisting of lower triangular $n$ by $n$ matrices, and $\rho$ the representation on the vector space $C^{n}$ defined by

$$
\rho(g) v=g \cdot v\left(g \in B_{n}^{-}, v={ }^{t}\left(v_{1}, \cdots, v_{n}\right) \in C^{n}\right) .
$$

Then the triplet $\left(B_{n}^{-} \times B_{n}, \tilde{\rho}_{1}, M(n, C)\right)$ is a P.V., and relative invariants and the $b$-function are known ([2], p. 150). In this case, Theorem 3.1 is true and we shall reduce the problem to this case.

For a polynomial $f(x)$ in $V$, we denote by $f^{*}(x)$ the polynomial defined in (2.2).

For any partition $\lambda=\left(\lambda_{1}, \cdots, \lambda_{n}\right)$, denoting by $\pi_{\lambda}$ the projection

$$
V_{\lambda_{1}} \otimes \cdots \otimes V_{\lambda_{n}} \longrightarrow V_{\lambda},
$$

we shall introduce two $G L(n, C)$-homomorphisms $\theta_{1}$ and $\theta_{2}$

$$
\Theta_{i} ; V_{\lambda} \otimes V_{\lambda^{\prime}} \longrightarrow V_{\lambda+\lambda^{\prime}} \quad(i=1,2),
$$

where $\lambda+\lambda^{\prime}$ denotes the partition $\left(\lambda_{1}+\lambda_{1}^{\prime}, \lambda_{2}+\lambda_{2}^{\prime}, \cdots, \lambda_{n}+\lambda_{n}^{\prime}\right)$.

For any $f$ in $V_{\lambda}$ and $f^{\prime}$ in $V_{\lambda^{\prime}}, \Theta_{i}\left(f \otimes f^{\prime}\right)$ are defined as follows;

$$
\Theta_{1}\left(f(x) \otimes f^{\prime}(x)\right)=f(x) \cdot f^{\prime}(x)
$$

and

$$
\Theta_{2}\left(f(x) \otimes f^{\prime}(x)\right)=\pi_{\lambda_{1}+\lambda_{n}}\left((\operatorname{det} x)^{\lambda_{1}} f^{*}(\operatorname{grad}) f^{\prime}(x)\right) .
$$

The decomposition of the $S L(n, C)$-module $V_{\lambda} \otimes V_{\lambda^{\prime}}$ into irreducible components contains $V_{\lambda+\lambda^{\prime}}$ with multiplicity one. The Schur's lemma says that $\Theta_{1}$ and $\Theta_{2}$ must be agree up to a constant.

On the other hand, a complete system of relative invariants of $\left(B_{n}^{-} \times B_{n}, \tilde{\rho}_{1}, M(n, C)\right)$ is given by $\left\{\Delta_{1}(x), \cdots, \Lambda_{n}(x)\right\}$ where

$$
\Delta_{i}=\operatorname{det}\left(\begin{array}{ccc}
x_{1}^{1} \cdots & x_{1}^{i} \\
\vdots & \vdots \\
x_{i}^{1} \cdots & x_{i}^{i}
\end{array}\right) \quad(1 \leq i \leq n) .
$$

For relative invariant polynomials $f$ and $f^{\prime}$ of the P.V. $\left(G \times G L\left(d_{1}, \cdots, d_{r}\right)\right.$, $\tilde{\rho}_{1}, M(n, C)$, let $\lambda$ and $\lambda^{\prime}$ denote the corresponding partitions, respectively.

Put, for $i=1,2, \cdots, n$,

$$
m_{i}=\lambda_{i}-\lambda_{i+1} \quad\left(\text { with } \lambda_{n+1}=0\right)
$$


and

$$
m_{i}^{\prime}=\lambda_{i}^{\prime}-\lambda_{i+1}^{\prime} \quad\left(\text { with } \lambda_{n+1}^{\prime}=0\right) .
$$

Then $\prod_{i=1}^{n} \Delta_{i}^{m_{i}}(x)$ and $\prod_{i=1}^{n} \Delta_{i}^{m_{i^{\prime}}}(x)$ are contained in $V_{\lambda}$ and $V_{\lambda^{\prime}}$, respectively. Therefore we have

$$
\frac{f^{*}(\mathrm{grad}) \cdot f^{\prime}(x)}{f(x) \cdot f^{\prime}(x)}=\frac{\prod_{i=1}^{n} \Delta_{i}^{*}(\mathrm{grad})^{m_{i}} \cdot \prod_{i=1}^{n} \Delta_{i}(x)^{m_{i^{\prime}}}}{\prod_{i=1}^{n} \Delta_{i}(x)^{m_{i}+m_{i^{\prime}}}}
$$

Thus we can reduce the proof to the case of the P.V. $\left(B_{n}^{-} \times B_{n}, \tilde{\rho}_{1}, M(n, C)\right)$, and we obtain (3.1). (3.2) is shown in a similar manner.

Q.E.D.

Corollary. Let $(G, \rho, V)$ be a P.V., $\left\{P_{1}, \cdots, P_{k}\right\}$ a complete system of relative invariants of $(G, \rho, V)$, and $d_{1}, \cdots, d_{k}$ degrees of $P_{1}, \cdots, P_{k}$, respectively. Then $\left(G \times G L(1, n-1), \tilde{\rho}_{1}, M(n, C)\right), n=\operatorname{dim} V$, is a P.V., and the $b$-functions $b_{x}(s)$ and $b_{x^{*}}(s)$ of $\left(G \times G L(1, n-1), \tilde{\rho}_{1}, M(n, C)\right.$ are given by

$$
b_{x}(s)=\frac{\gamma(s)}{\gamma(s-\delta(\chi))} \quad \text { and } \quad b_{x}^{*}(s)=\frac{\gamma^{*}(s)}{\gamma^{*}\left(s-\delta^{*}(\chi)\right)}
$$

where

$$
\gamma(s)=\Gamma\left(s_{0}+d_{1} s_{1}+\cdots+d_{k} s_{k}+n\right) \Gamma\left(s_{0}+n-1\right) \cdots \Gamma\left(s_{0}+1\right)
$$

and

$$
\begin{gathered}
\gamma^{*}(s)=\Gamma\left(s_{0}+d_{1} s_{1}+\cdots+d_{k} s_{k}+n\right) \Gamma\left(s_{0}+d_{1} s_{1}+\cdots+d_{k} s_{k}+n-1\right) \\
\cdots \Gamma\left(s_{0}+d_{1} s_{1}+\cdots+d_{k} s_{k}+2\right) \Gamma\left(s_{0}+1\right) .
\end{gathered}
$$

$\S 4$. Prehomogeneous vector spaces $\left(G \times B_{n}, \tilde{\rho}_{1}, M(n, C)\right)$

Let $G$ be a connected linear semi-simple algebraic group $(G \neq\{e\})$, $\rho: G \rightarrow G L(V)$ an $n$-dimensional irreducible representation, all defined over C. Let $\left(G \times B_{n}, \tilde{\rho}_{1}, M(n, C)\right)$ be a P.V. Then, $\operatorname{dim}\left(G \times B_{n}\right) \geq \operatorname{dim} M(n, C)$, and hence we have:

$$
\operatorname{dim} G \geq \frac{1}{2} n(n-1)
$$

Since $G$ is semi-simple, we may assume that a triplet $(G, \rho, V)$ is of the form: 


$$
\begin{aligned}
& G=G_{1} \times G_{2} \times \cdots \times G_{k}, \\
& \rho=\rho_{1} \otimes \rho_{2} \otimes \cdots \otimes \rho_{k},
\end{aligned}
$$

and

$$
V=V\left(d_{1}\right) \otimes V\left(d_{2}\right) \otimes \cdots \otimes V\left(d_{k}\right) \text { with } \quad d_{1} \geq d_{2} \geq \cdots \geq d_{k} \geq 2,
$$

where each $G_{i}$ is a connected simple algebraic group, $\rho_{i}$ is an irreducible representations of $G_{i}$ on the $d_{i}$-dimensional $C$-vector space $V\left(d_{i}\right)(1 \leq i \leq k)$. Therefore if $\left(G \times B_{n}, \tilde{\rho}_{1}, M(n, C)\right)$ is a P.V. we have

$$
\sum_{i=1}^{k} \operatorname{dim} G_{i} \geq \frac{1}{2} d_{1} \cdots d_{k} \quad\left(d_{1} \cdots d_{k}-1\right) .
$$

Lemma 4.1. Assume that a triplet $\left(G \times B_{n}, \tilde{\rho}_{1}, M(n, C)\right)$ is a P.V. Then we have $k=1$ or $(G, \rho, V)=(S L(2) \times S L(2), \square \otimes \square, V(4))$.

Proof. The image $\rho_{i}\left(G_{i}\right)$ of the simple algebraic group $G_{i}$ is contained in $S L\left(d_{i}\right)$. By (4.1), we have

$$
\sum_{i=1}^{k}\left(d_{i}^{2}-1\right) \geq \frac{1}{2} d_{1} \cdots d_{k} \quad\left(d_{1} \cdots d_{k}-1\right) .
$$

If $k \geq 3$, this inequality implies that

$$
1>d_{1}^{2}\left(2^{2 k-3}-2^{k-2}-k\right)+k .
$$

It is easy to show that $2^{2 k-3}>2^{k-2}+k$ for $k \geq 3$. This is impossible and hence we have $k \leq 2$. When $k=2$, we have $d_{1}^{2}+d_{2}^{2}>\frac{1}{2} d_{1} d_{2}\left(d_{1} d_{2}-1\right)$. This inequality implies that $d_{1}=d_{2}=2$.

The following lemma can be easily proved.

Lemma 4.2. Let $G$ be a semi-simple linear algebraic group and $\rho: G$ $\rightarrow G L(V)$ be an irreducible representation on an $n$-dimensional vector space $V(n)$ satisfying

$$
\operatorname{dim} G \geq \frac{1}{2} n(n-1)
$$

Then $(G, \rho, V(n))$ is one of:

(1) $(S L(n), \square, V(n))$

(2) $(S L(2), \square, V(3))$

( 3 ) $(S L(4),-V(6))$

(4) $(S L(2) \times S L(2), \square \otimes \square, V(4))$ 
(5) $(S p(m), \square, V(n))(n=2 m)$

(6) $\quad(S O(n), \square, V(n))(n \geq 3)$

( 7 ) $(S p(2),-, V(5))$

(for classical group, we write the Young diagram corresponding to $\rho$ ).

The image of the representation of $S p(2)$ is $S O(5)$ and the kernel is $\{ \pm 1\}$. Therefore we may identify the triplet $(S p(2),-V(5))$ with the triplet $(S O(5), \square, V(5))$. Similarly, we identify the triplets $(2) \sim(4)$ with the triplet (6), $n=3,6,4$, respectively.

Case 1. $(G, \rho, V)=(S L(n), \square, V(n))$.

The triplet $(S L(n), \tilde{\square}, M(n, C))$ is a trivial P.V. (See [2], and the singular set $S$ is given by

$$
S=\{X \in M(n, C) ; \operatorname{det} X=0\} .
$$

The Young diagram corresponding to the relative invariant $\operatorname{det} X$ is

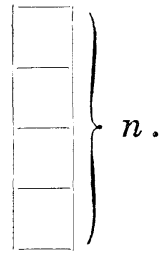

By Theorem 1, the $b$-function is given by

$$
b(s)=s(s+1) \cdots(s+n-1) .
$$

Remark. The $b$-function of $\operatorname{det} X$ is well known (See [2])

Case 2. $(G, \rho, V)=(S O(n), \square, V(n))$.

The triplet $(S O(n), \tilde{\square}, M(n, C))$ is a P.V., and the $b$-function of it is known (See [2]).

We shall determine the $b$-function by Theorem 3.1 .

For a $x=\left[x^{1}, \cdots, x^{n}\right] \in M(n, C)$, put

$$
P_{i}(x)=\operatorname{det}\left(\begin{array}{cc}
\left(x^{1}, x^{1}\right), \cdots,\left(x^{1}, x^{n}\right) \\
\vdots & \vdots \\
\left(x^{n}, x^{1}\right), \cdots,\left(x^{n}, x^{n}\right)
\end{array}\right) \quad(1 \leq i \leq n-1)
$$

where $\left(x^{k}, x^{l}\right)={ }^{t} x^{k} \cdot x^{l}$, and $P_{0}(x)=\operatorname{det} x$. 
Then the singular set $S$ is $S_{0} \cup \ldots \cup S_{n-1}$ with

$$
S_{i}=\left\{x \in M(n, C) ; P_{i}(x)=0\right\} \quad(0 \leq i \leq n-1) .
$$

Thus the Young diagrams corresponding to relative invariants $P_{1}, \cdots, P_{n}$ ， and $P_{0}$ are, respectively,

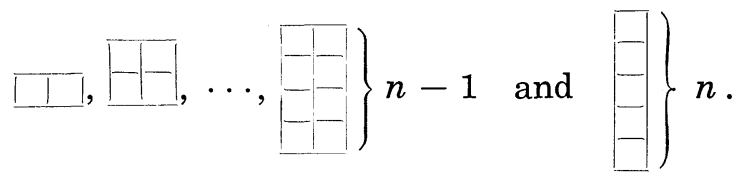

By Theorem 1, we have:

$$
b_{\chi}(s)=\gamma(s) / \gamma(s-\delta \chi)
$$

where

$$
\begin{aligned}
\gamma(s)= & \Gamma\left(s_{0}+2 s_{1}+\cdots+2 s_{n-1}+n\right) \\
& \times \Gamma\left(s_{0}+2 s_{2}+\cdots+2 s_{n-1}+n-1\right) \cdots \Gamma\left(s_{0}+1\right) .
\end{aligned}
$$

Case 3. $(G, \rho, V)=(S p(m), \square V(n)) \quad(n=2 m)$

Denote by $[x, y]$ the skew symmetric bilinear form on $V(n) \times V(n)$ defined as follows.

$$
\begin{aligned}
& {[x, y]=x_{1} y_{1}^{\prime}-x_{1}^{\prime} y_{1}+\cdots+x_{m} y_{m}^{\prime}-x_{m}^{\prime} y_{m}} \\
& \quad \text { with } x={ }^{t}\left(x_{1}, x_{1}^{\prime}, \cdots, x_{m}, x_{m}^{\prime}\right) \text { and } y={ }^{t}\left(y_{1}, y_{1}^{\prime}, \cdots, y_{m}, y_{m}^{\prime}\right) .
\end{aligned}
$$

For $x=\left(x^{1}, \cdots, x^{n}\right) \in M(n, C)$. Put

$$
P_{k}(x)=\operatorname{Pff}\left(\left[x^{i}, x^{j}\right]\right)_{\substack{1 \leq i \leq 2 k \\ 1 \leq j \leq 2 k}}(1 \leq k \leq m-1),
$$

and

$$
P_{0}(x)=\operatorname{det}(x)
$$

where Pff denotes the Pfaffian.

It is easy to show that, if a point $x_{0}$ of $M(n, C)$ satisfies $\prod_{i=0}^{m-1} P_{i}(x)$ $\neq 0$, there exists a $(g, T) \in S p(m) \times B_{n}$ such that $g x_{0} T^{-1}=1_{n}$. Therefore the triplet $(S p(m), \square, M(n, C))$ is a P.V., and the singular set $S$ is

$$
\begin{aligned}
& S=S_{0} \cup S_{1} \cup \cdots \cup S_{m} \\
& \quad \text { with } \quad S_{i}=\left\{X \in M(n, C) \mid P_{i}(x)=0\right\} \quad(0 \leq i \leq m) .
\end{aligned}
$$

The Young diagrams corresponding to relative invariants $P_{1}, \cdots, P_{m-1}$ and $P_{0}$ are, respectively, 


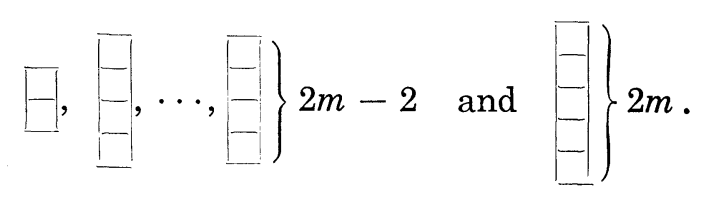

Put

$$
\begin{aligned}
\gamma(s)= & \Gamma\left(s_{0}+s_{1}+\cdots+s_{m-1}+n\right) \Gamma\left(s_{0}+s_{1}+\cdots+s_{m-1}+n-1\right) \\
& \times \Gamma\left(s_{0}+s_{2}+\cdots+s_{m-1}+n-2\right) \Gamma\left(s_{0}+s_{2}+\cdots+s_{m-1}+n-3\right) \\
& \times \Gamma\left(s_{0}+s_{m-1}+4\right) \Gamma\left(s_{0}+s_{m-1}+3\right) \\
& \times \Gamma\left(s_{0}+2\right) \cdot \Gamma\left(s_{0}+1\right) .
\end{aligned}
$$

Then, by Theorem 1 , the $b$-function $b_{x}(s)$ is given by

$$
b_{\chi}(s)=\gamma(s) / \gamma(s-\delta(\chi)) .
$$

Now we obtain the following theorem.

Theorem 4.1. Let $\left(G \times B_{n}, \tilde{\rho}_{1}, M(n, C)\right)$ be a P.V., where $G$ is a semisimple connected linear algebraic group, $B_{n}$ the group consists of all $n \times n$ complex triangular matrix, $\rho: G \rightarrow G L(V)$ an irreducible representation on an $n$-dimensional vector space, all defined over $C$. Let $\left\{P_{0}, \cdots, P_{k}\right\}$ be a complete set of irreducible relative invariants of $\left(G \times B_{n}, \tilde{\rho}_{1}, M(n, C)\right)$. Then $(G, \rho, V)$ is one of the following P.V.'s,

(1) $(S L(n), \square, V(n))$.

(i) $k=0$

(ii) $\gamma(s)=\Gamma\left(s_{0}+n\right) \cdots \Gamma\left(s_{0}+1\right)$

(iii) $\gamma^{*}(s)=\Gamma\left(s_{0}+n\right) \cdots \Gamma\left(s_{0}+1\right)$.

(2) $(S p(m), \square, V(n)) \quad(n=2 m)$.

(i) $k=m-1$

(ii) $\gamma(s)=\Gamma\left(s_{0}+s_{1}+\cdots+s_{m-1}-n\right) \Gamma\left(s_{0}+s_{1}+\cdots+s_{m-1}+n-1\right)$

$\times \Gamma\left(s_{0}+s_{2}+\cdots+s_{m-2}+n-2\right)$

$\times \Gamma\left(s_{0}+s_{2}+\cdots+s_{m-1}+n-3\right)$

$\times \Gamma\left(s_{0}+s_{m-1}+4\right) \Gamma\left(s_{0}+s_{m-1}+3\right) \Gamma\left(s_{0}+2\right) \Gamma\left(s_{0}+1\right)$.

(iii) $\gamma^{*}(s)=\Gamma\left(s_{0}+s_{1}+\cdots+s_{m-1}+n\right) \Gamma\left(s_{0}+s_{1}+\cdots+s_{m-1}+n-1\right)$

$\times \Gamma\left(s_{0}+s_{1}+\cdots+s_{m-2}+n-3\right)$

$\times \Gamma\left(s_{0}+s_{1}+\cdots+s_{m-2}+n-4\right)$

$\times \Gamma\left(s_{0}+s_{1}+4\right) \Gamma\left(s_{0}+s_{1}+3\right) \Gamma\left(s_{0}+2\right) \Gamma\left(s_{0}+1\right)$. 
(3) $(S O(n), \square, V(n))$.

(i) $k=n-1$

(ii) $\gamma(s)=\Gamma\left(s_{0}+2 s_{1}+\cdots+2 s_{n-1}+n\right)$

$$
\times \Gamma\left(s_{0}+2 s_{2}+\cdots+2 s_{n-1}+n-1\right)
$$

$\times \Gamma\left(s_{0}+2 s_{3}+\cdots+2 s_{n-1}+n-2\right)$

$\times \Gamma\left(s_{0}+2 s_{4}+\cdots+2 s_{n-1}+n-3\right)$

$\times \Gamma\left(s_{0}+2 s_{n-1}+2\right) \Gamma\left(s_{0}+1\right)$.

(iii) $\gamma^{*}(s)=\Gamma\left(s_{0}+2 s_{1}+\cdots+2 s_{n-1}+n\right)$

$\times \Gamma\left(s_{0}+2 s_{1}+\cdots+2 s_{n-2}+n-1\right)$

$\times \Gamma\left(s_{0}+2 s_{1}+\cdots+2 s_{n-3}+n-2\right)$

$\times \Gamma\left(s_{0}+2 s_{1}+\cdots+2 s_{n-4}+n-3\right)$

$\times \Gamma\left(s_{0}+2 s_{1}+2\right) \Gamma\left(s_{0}+1\right)$.

In the next article, we shall be concerned with zeta-functions associated with prehomogeneous vector spaces $\left(G \times G L\left(d_{1}, \cdots, d_{r}\right), \tilde{\rho}_{1}, M(n, C)\right)$.

\section{REFERENCES}

[1] H. Weyl, Classical Groups, Princeton University Press, 1964.

[2] M. Sato, Theory of prehomogeneous vector space (note by T. Shintani in Japanese), Sugaku no ayumi, 15 (1970), 85-157.

[ 3 ] M. Sato and T. Kimura, A classification of Irreducible Prehomogeneous Vector Spaces and Their Relative Invariants, Nagoya Math. J., 65 (1977), 1-155.

Department of Mathematics

Faculty of Science

Chikusa-ku, Nagoya 464

Japan 